EPJ Web of Conferences 114, 02048 (2016)

DOI: $10.1051 /$ epjconf/201611402048

(C) Owned by the authors, published by EDP Sciences, 2016

\title{
On the interaction between turbulence grids and boundary layers
}

\author{
Thomas Irps, Vasudevan Kanjirakkad \\ Thermo-Fluid Mechanics Research Centre, University of Sussex, Brighton, BN1 9QT, United Kingdom
}

\begin{abstract}
Turbulence grids are widely used in wind tunnels to produce representative turbulence levels when testing aerodynamic phenomena around models. Although the purpose of the grid is to introduce a desired turbulence level in the freestream flow, the wall boundary layers of the tunnel are subjected to modification due to the presence of such grids. This could have major implications to the flow around the models to be tested and hence there is a need to further understand this interaction. The study described in this paper examines wind tunnel wall boundary layer modification by turbulence grids of different mesh sizes and porosities to understand the effect of these parameters on such interaction. Experimental results are presented in the form of pressure loss coefficients, boundary layer velocity profiles and the statistics of turbulence modification.
\end{abstract}

\section{Introduction}

Grids of many shapes (round, square, structured, fractal, etc.), fabrication types (woven, welded/braced, perforated) and working principle (static, agitated/ pulsed, jet driven, active and passive) have been extensively used by aerodynamicists to introduce representative flow perturbations and turbulence into wind tunnel test sections. Since the turbulence produced by the grid decays with the flow downstream, the position of the grid installation becomes important. In many occasions this means that the grid needs to be positioned in relative proximity to the test section and the model. When the aerodynamic model to be tested is suspended away from the side walls of the test section, these grids are effective in providing a certain mainstream turbulence level with a uniform mean velocity profile in the transverse directions. However there are many test cases where the models to be tested need to be in close proximity to the wind tunnel walls (e.g., study of ground effects on moving objects, wind loading of static objects/buildings at ground level, turbomachinery blade cascade tests etc.). An interesting issue that is less discussed when one is encountered with the latter scenario is the interaction of the wind tunnel wall boundary layer upstream of the grid with the grid structure itself. Such interaction could produce unwanted localised flow variations both in an instantaneous and time averaged sense that could extend for an appreciable distance downstream and could affect the test conditions if the model to be tested is in close proximity.

Although only a handful of authors have discussed this in published literature, a common observation from those who did is the existence of a downstream velocity 'overshoot' and a thinning of the boundary layer over the wall surface that intersects the grid structure. To the authors' knowledge, the first observation of the boundary layer overshoot was described by Owen et al. [1] in an experiment which featured non-linearly distributed parallel rod grids. In that study which focused on the production of uniform shear flow, the authors describe the occurrence of local acceleration of the flow (relative to the mainstream flow) due to the pressure drop across the grid. This is explained by stating that the boundary layer suffers a lower total pressure loss due to the slower velocities encountered within it. Lau et al. [2] demonstrated the same effect and used the energy equation to show that a pressure drop coefficient larger than unity would create an overshoot with increasing size proportional to an increasing pressure drop. Mehta [3] suggested that the magnitude of the pressure drop coefficient (which thins the boundary layer) and the screen rigidity (i.e. possible curvature of the screen) plays a larger role in the generation of the overshoot due to the maximum streamline inclination towards the wind-tunnel surface.

When studying turbulence grid design it is important to understand the major parameters of relevance and how to use them to characterise the grid. Roach [4] provided a thorough summary of different geometric parameters that characterise a static turbulence grid (i.e. not featuring agitated bars, jet, aerofoil cascades and others). As shown by Mehta [3] and Roach [4] it is not the porosity alone that affects the performance of the flow but rather the imperfections and geometric parameters such as the grid type and curvature effects. 
Kurian et al. [5] investigated flow through five different grids with the same porosity to characterize grid generated turbulence with a focus on energy spectra, correlation functions, turbulence length-scales, energy dissipation rates among others. The authors note that porosities should always be larger than 0.55 in order to avoid large-scale mean velocity profile fluctuations due to the merging of adjacent jets emanating from the holes.

Our intention in the on-going work, part of which is presented here in this paper, is to investigate the occurrence of the boundary layer overshoot, the extent and nature of its spatial existence and the cause of its origin. The paper tries to provide some insight into the boundary layer modification and the overshoot effect as brought about by the turbulence grid using the pressure drop data measured across the grid, velocity profiles resulting from the grid and the observed turbulence statistics upstream and downstream of the grid.

\section{Methodology}

Grid selection: To generate the overshoot five grids are employed in this study, three of which have the same porosity but varying mesh sizes and three having relatively similar mesh sizes but significantly varying porosities. These are manufactured from $2 \mathrm{~mm}$ thick aluminium sheets and are made following the design criteria described in Roach [4] for square-mesh arrays of square bars (SMS). All of the selected grids are expected to produce a pressure drop coefficient $(K)$ larger than unity as per Roach's correlations. These employ empirically determined variables $A$ and $B$ in the form of

$$
K=\frac{\Delta p}{q}=A\left(\frac{1}{\beta^{2}}-1\right)^{B},
$$

where $A$ is 0.98 and $B$ is 1.09 and $q$ is the upstream reference dynamic pressure

$$
q=\frac{1}{2} \rho U_{r e f}^{2}
$$

The porosity is determined through

$$
\beta=\left(1-\frac{d}{M}\right)^{2}
$$

where, $d$ is the strut width and $M$ the mesh height (which is also the unit size). The resulting mesh characteristics for the five chosen grids (hereafter designated as G1, G2 G3, G4 and G5) are listed in Table 1.

Table 1. Grid parameters.

\begin{tabular}{|l|l|l|l|l|l|}
\hline Grid & \multicolumn{1}{|c|}{ G1 } & \multicolumn{1}{|c|}{ G2 } & \multicolumn{1}{|c|}{ G3 } & \multicolumn{1}{|c|}{ G4 } & \multicolumn{1}{|c|}{ G5 } \\
\hline Hole (mm) & 4.5 & 8 & 9 & 10 & 18 \\
\hline Strut (mm) & 1.5 & 4 & 3 & 2 & 6 \\
\hline Unit size (mm) & 6 & 12 & 12 & 12 & 24 \\
\hline Porosity & 0.563 & 0.444 & 0.563 & 0.694 & 0.563 \\
\hline K & 2.27 & 4.51 & 2.27 & 1.05 & 2.27 \\
\hline
\end{tabular}

Experimental setup and instrumentation: The test section is incorporated into the Sussex low-speed wind-tunnel which is a blower type tunnel driven by a centrifugal blower, handling a top range of 18.2 HP and 997 RPM with a resulting maximum flow rate of $6.32 \mathrm{~m}^{3} / \mathrm{s}$. The flow through the tunnel is controlled via a mechanically regulated throttle at the inlet of the blower. Following the centrifugal blower is a diffuser section which consists of four screens. This is followed by a contraction section and the test section which is $2.2 \mathrm{~m}$ long and has a crosssectional dimension of $0.15 \mathrm{~m} \times 0.456 \mathrm{~m}$.

Flow and boundary layer development is only measured along the lower horizontal wall of the duct and measurements are carried out only along the centreline of the duct thereby minimising the effects due to the sidewalls.

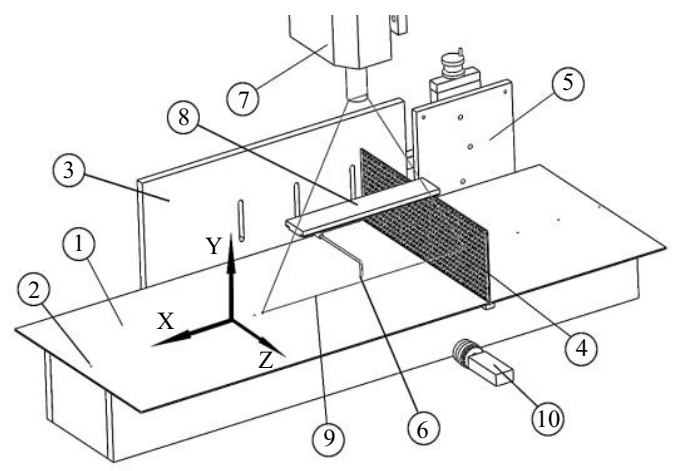

Figure 1. Test section instrumentation.

As shown in Figure 1 the setup consists of the fixed surface lower wall (1) where boundary layer flow is tracked. A series of static pressure tappings (2) are used along the centre line of the lower wall to measure pressure drop along the duct. A window (3) with multiple vertical slots is used to allow hot-wire (6) and pneumatic probe traverses in the wall normal direction (y-axis) at selected locations (see Figure 2) upstream and downstream of the grid (4). The linear actuator mechanism for traversing the probe is fixed on the outside of the wind-tunnel (5). The non-intrusive PIV setup consists of the non-reflective glass (8), the laser head (7), and the camera (10) for visualising the intended measuring area (9). The grids are held firmly in place by two u-shaped profiles that are cut into the upper and lower walls of the duct. It is important to note that care was taken to avoid close proximity between the lowest horizontal bar of the grid and the lower surface of the duct in order to prevent it from acting as an undesired boundary layer trip.
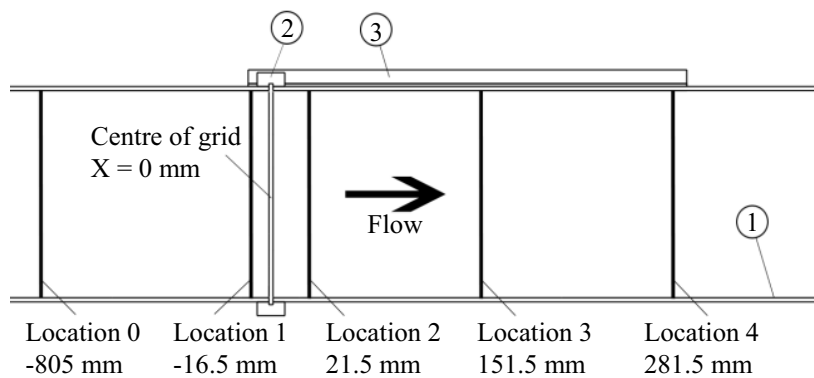

Figure 2. Measurement locations (not to scale).

Four measurement locations are chosen for gathering boundary layer data as shown in Figure 2. These locations allow to track both the flow development downstream of the grid as well as the boundary layer profile entering the 
grid. The number and the distance of the measurement locations with respect to that of the grid is also shown. In Figure 2, (1) denotes the test-section surface, (2) the junction securing grid in place and (3) the non-reflective glass for PIV measurements.

The various instrumentation types employed in this study include; hotwire (CTA), particle image velocimetry (PIV) and a range of steady pneumatic pressure measurements (for static and total pressure information).

The CTA setup consists of a single-wire SSP11 1-D all-purpose probe ( $5 \mu \mathrm{m}$ diameter), held by a $90^{\circ}$ support of the SSH21 type. A 4m long cable (A1863) connects the probe to the control system which is a Streamline 90 N10 CTA frame from Dantec. The output from this is connected through NI USB-6251 A/D board to a laptop (which is configured via the Streamware 3.4 software) where data is logged using a LabView based software. All CTA signals were obtained at a frequency of $20 \mathrm{kHz}$.

The PIV laser system consists of a New Wave Research Class IV Solo PIV $120 \mathrm{Nd}$ :Yag laser source. The beam diameter is $4.5 \mathrm{~mm}$ and has a pulse width of 3-5 ns. In order to generate sufficient amount of seeding particles the smoke machine "Le Maitre G-Force 1" is used which can generate up to $320 \mathrm{~m}^{3} / \mathrm{min}$ of smoke with a particle diameter less than $5 \mu \mathrm{m}$. The camera used to capture the seeding is the Dantec Dynamics Flowsense Charged Coupled Device (CCD) camera with a resolution of 1600 $\mathrm{x} 1186$ pixels and has a 10 bit analogue to digital converter. These two systems are linked to the Dantec Dynamics FlowMap processor which contains a correlator unit, input buffers and the synchronisation unit. The PIV measurements are currently ongoing and therefore only a limited amount of information would be presented in this paper.

The tunnel velocity is set using a pitot-static probe placed at mid height along the centre line of the duct 805 $\mathrm{mm}$ upstream of the turbulence grid, which is also used as reference $\left(U_{\text {ref }}\right)$ velocity for all purposes. The above pitotstatic tube is connected to a FC-034 $500 \mathrm{~Pa}$ differential pressure transducer. A Druck DPI 740 barometer is used to measure the ambient pressure. A commercial thermocouple reader accurate to within $\pm 1^{\circ} \mathrm{C}$ is used to track variation in room temperatures during the measurement. The wall static pressures along the duct lower surface and the total pressure data from a flattened pitot tube were captured using a 16 channel DSA 3217 transducer array. Tests were conducted for two different freestream velocities; $10.6 \mathrm{~m} / \mathrm{s}$ and $17.6 \mathrm{~m} / \mathrm{s}$. However the results are only shown for the lower velocity case since initial analysis has produced very similar outcomes.

\section{Results}

In order to characterize the mean flow and the boundary layers several flow parameter definitions are used in this paper. To evaluate the static pressure variation inside the wind tunnel a static pressure coefficient is used as defined by:

$$
C_{s p}=\frac{p_{i}}{q_{r e f}}
$$

where, $p_{i}$ is the local static pressure (gauge) along the test section and $q_{\text {ref }}$ is the dynamic pressure of the flow at the reference location. The magnitude of $C_{s p}$ is comparable to that of the grid pressure drop coefficient $(K)$ as defined by Roach [4].

For the unperturbed velocity profiles, the boundary layer height $\left(\delta_{99}\right)$ is calculated as wall normal distance where the velocity reaches $99 \%$ of that in the freestream $\left(U_{\infty}\right)$. For cases with an overshoot the boundary layer height is calculated as the height at which the velocity reached $99 \%$ of the overshoot maxima.

The grid turbulence intensity has been empirically determined by Roach [4] to follow a decay law as represented by

$$
T u=C\left(\frac{x}{d}\right)^{-\frac{5}{7}},
$$

where, the value of the constant $\mathrm{C}$ for grids of the SMS type is 1.13

The measured turbulence intensity $(T u)$ is determined using the root mean square of the fluctuating components $(u)$ and the mean freestream velocity $(U)$ as per the expression,

$$
T u=\frac{(u)_{r m s}}{U} .
$$

For defining the turbulent length-scale $\left(\lambda_{x}\right)$ we only consider the streamwise components of the velocity fluctuations and the derived energy spectrum $E(f)$ :

$$
\lambda_{x}=\left[\frac{E(f) U}{4 \overline{u^{2}}}\right]_{f \rightarrow 0} .
$$

As explained by Roach [4] and by El-Gabry et al. [6] the frequency content above $10 \mathrm{kHz}$ is negligible and only the energy content of the data asymptotically approaching a fixed value at frequencies close to zero is employed. This correlation assumes that the turbulent flow is truly homogenous and isotropic. The energy spectral density, $E(f)$, for a given frequency band as defined by:

$$
E(f)=\frac{F F T(u)^{2} / L}{S}
$$

where, $L$ is the sample length and $S$ the sampling frequency.

To illustrate the boundary layer development through the duct and the interaction effect with the turbulence grid that is placed in the flow, Figure 3 compares the velocity profiles (hotwire) with and without a grid at four different locations along the duct on either side of the grid. Grid 3 (G3) is used as an example. Figure 3A compares the profiles at location-1 which is marginally upstream of the grid. Although this is an upstream location, it is clear that this is well within the potential field of the grid and hence there is a clear modification of the flow visible in the form of a thinning of the boundary layer when the grid is present as quantified in Table 3 . 

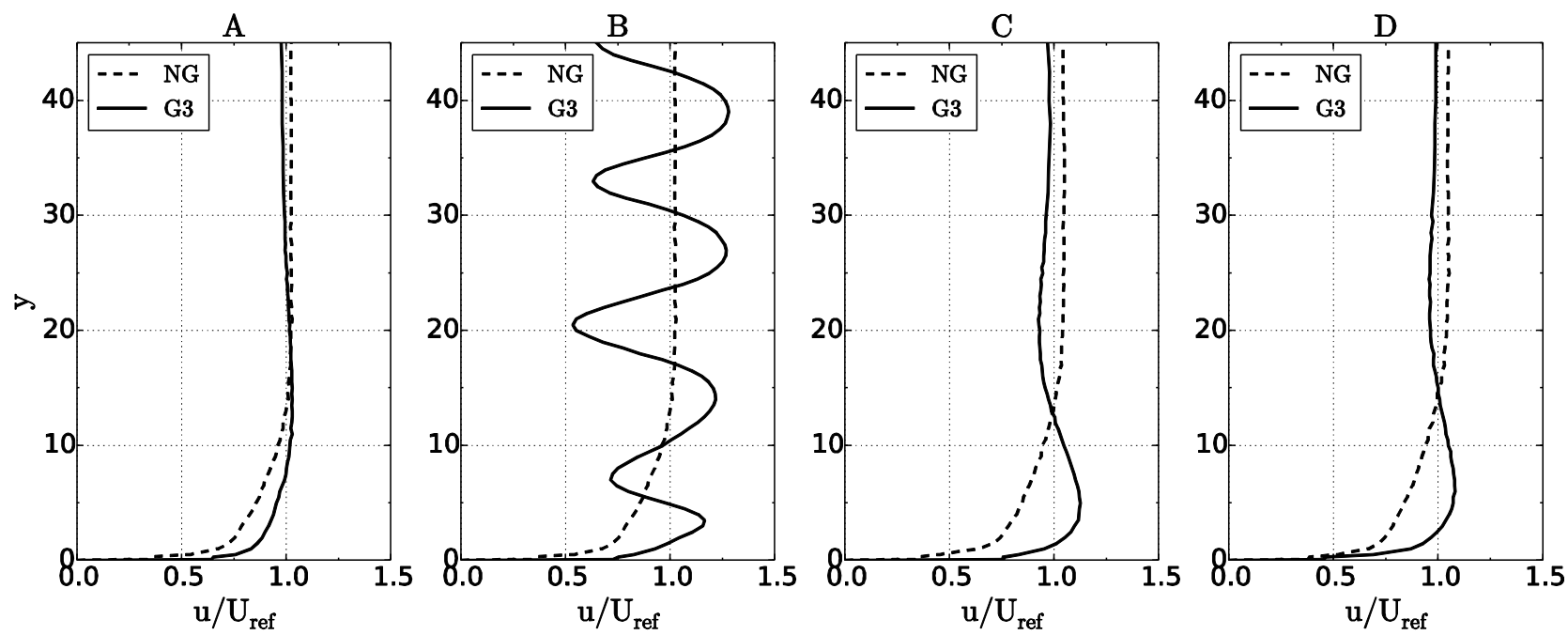

Figure 3. Boundary layer modification through grid interaction. A, B, C and D correspond to locations 1, 2, 3 and 4 respectively.

Figure 3B shows that at location 2 that is immediately downstream of the grid a more dramatic velocity distribution, where one cannot clearly discern the boundary layer due to the alternate wake and jet pattern, is emerging from the grid. Locations 3 and 4 are sufficiently downstream of the grid that the individual jets have now mixed and merged with one another but leaving a bump in the profile near the wall which is referred to as the velocity overshoot. Although the overshoot is present at both location-3 and location-4, it is clear from Figure 3 that the magnitude of the overshoot reduces as the flow marches downstream and the location of the velocity maxima moves further away from the wall. It is expected that the overshoot would disappear further downstream and a cleaner profile would result. However, it remains a fact that, turbulence intensities get damped and length-scales get larger further downstream. In many wind tunnel test setups this could mean that the model to be tested needs to be placed within the region of an active overshoot (for meeting a prescribed intensity and length-scale criteria) thus altering the 'ideal' test condition that was intended.

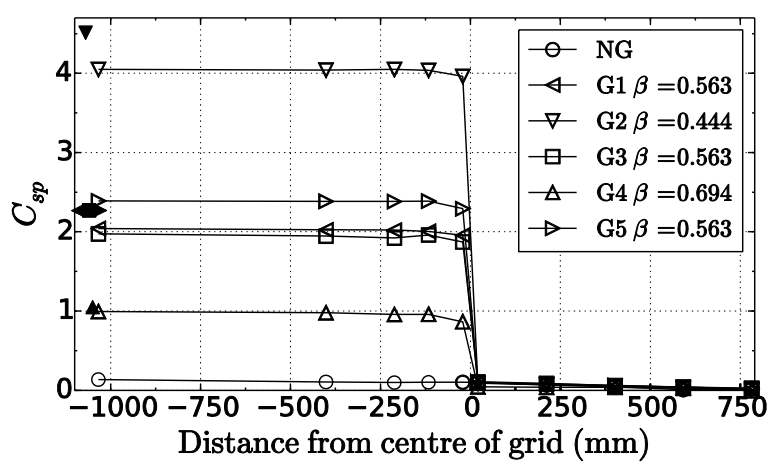

Figure 4. Wall static pressure coefficient variation in the test section.

One of the main consequences of placing a turbulence grid in the flow is the pressure drop that results due to the loss generated by the grid. The wall static pressure coefficient $\left(C_{s p}\right)$ is presented in Figure 4 , which shows the pressure drop effected by the placement of the grid in an otherwise clean duct test section (designated as NG, referring to a no-grid condition). Shown also alongside in closed symbols (of the same type) is the value of the pressure drop coefficient $(K)$ for the SMS type grid from Roach's correlations. It is clear that the measurements largely agree with the results from the correlation. The slight disagreement as seen between grids of the same porosity (G1, G3 and G5) is more likely to be a result of manufacturing imperfections. It is possible that the coefficient of discharge of individual mesh openings could have been altered due to locally rounded edges of the strut. Otherwise these three grids exhibit similar pressure drops as expected.

Figure 5 presents the results from boundary layer traverses obtained with and without a grid at the four traverse locations for all five grids tested. As discussed previously, the flow immediately in front of all four grids (location-1) undergo a modification due to the potential interaction with the grid as seen in Figure 5A. The general tendency is to produce a thinner boundary layer, when a grid is present, as can be seen from the measured boundary layer thicknesses before (location-1) and after (location-2) the grid in Table 3. A small 'waviness' is also present on all of the profiles at location-1. This modification is thought to be related to the deceleration of the streamlines in the presence of the grid, but this needs to be understood further. Figure 5B shows the wall normal velocity distribution at location-2 which is immediately downstream of the grids and therefore strongly influenced by the alternate jets and wakes emerging from the openings and struts, respectively, of the grid. When compared with the no-grid case, it can be observed that the amplitude of the sinusoidal pattern is strongly linked to the mesh porosity such that the large amplitudes are associated with lower porosities. The strong local variations make the calculation of boundary layer integral parameters very difficult and hard to interpret at this location. 

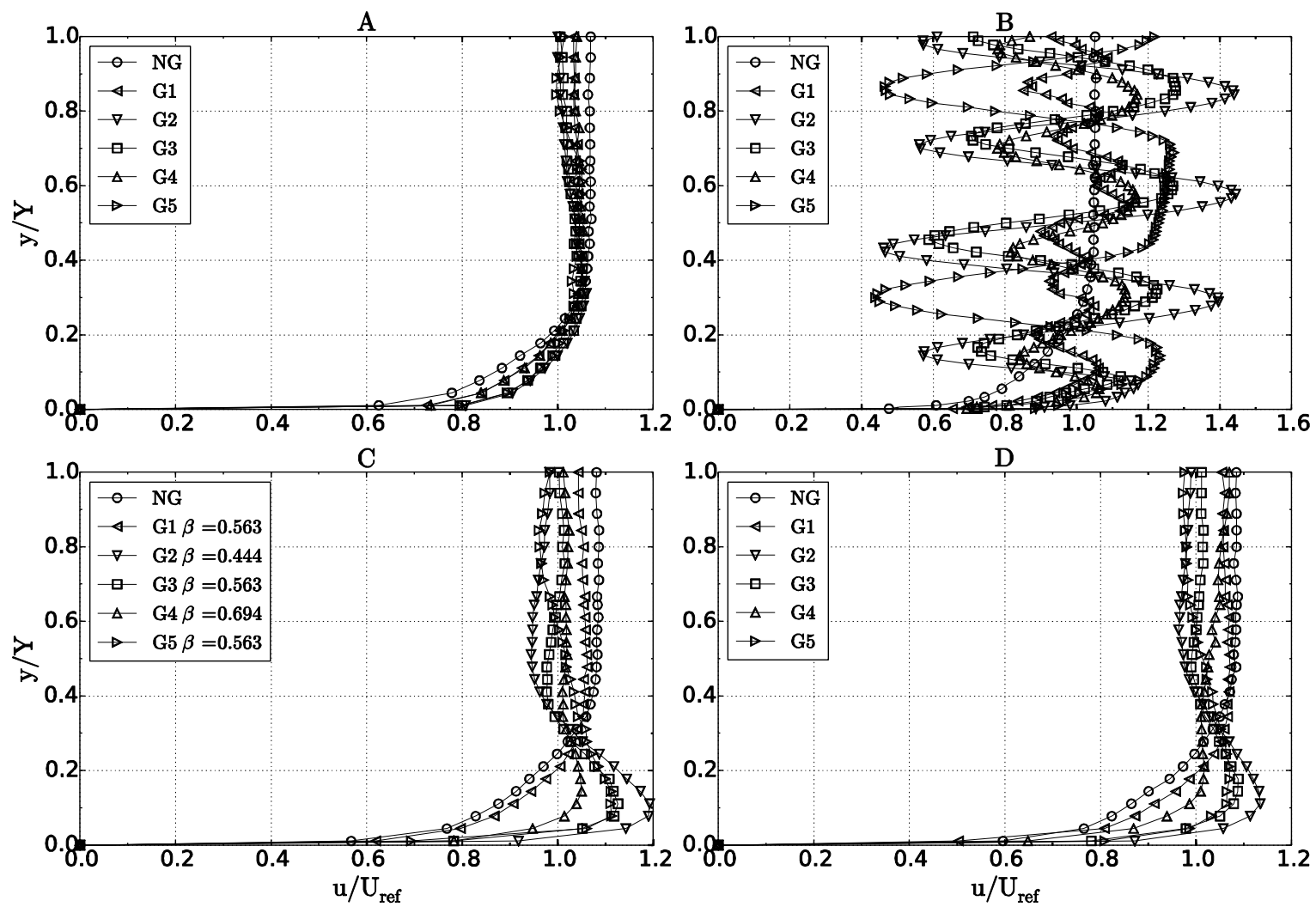

Figure 5. Boundary layer velocity profiles using hotwire measurements from the various traverse locations. Figures A, B, C and correspond to traverse locations 1, 2, 3 and 4 respectively. Here $\mathrm{Y}$ corresponds to $\mathrm{y}=45 \mathrm{~mm}$ which is the last traverse coordinate normal to the surface.

Figure $5 \mathrm{C}$ and $5 \mathrm{D}$ show the velocity profiles further downstream of the grid at locations 3 and 4 respectively. At both locations the wake/jet related fluctuations (as seen at location-2) are absent due to the strong mixing and diffusion caused by the turbulent flow, which in turn is produced at the individual jet boundaries and from their subsequent merger with each other. The most notable feature of the flow in these locations is the lobbed structure closer to the wall that signifies the velocity overshoot as has been mentioned earlier in the paper. Similar to the sinusoidal variations in velocity, as found at location- 2 , the magnitude of the overshoot seems to be an inverse function of the grid porosity. The overshoot is larger for grids with lower porosities as seen by comparing those produced by grids G2, G3 and G4 in Figures 5C and 5D. When the mesh size is varied with a fixed porosity the overshoot is seen to increase first with the mesh size and then reduce afterwards as seen for the grids G1, G3 and G5 in Figures $5 \mathrm{C}$ and 5D. This suggests that, for a fixed porosity case, there is an 'optimum' mesh size for which the overshoot is maximised. Mehta's explanation of the overshoot as a simple function of the differential pressure loss effect across the incoming boundary layer seems to be inadequate to explain the actual physical mechanism that causes the formation of the overshoot since grids with relatively similar pressure drops are seen to produce dissimilar overshoots. The time-accurate and timeaveraged data from the hotwire and PIV measurements during the present investigations will be further analysed to understand this phenomena better at a later stage when the PIV results are fully available. Note also from Figures $5 \mathrm{C}$ and $5 \mathrm{D}$ that an appreciable 'waviness' is present in the profiles in the wall normal direction at location-3 and location-4. This was also observed by Mehta [3] and was reiterated by Kurian [5] and has been explained to be associated with the random merging of adjacent jets amalgamating to form longitudinal vortices. In the present experiments this large scale non-uniformity is seen to get smoothened but not entirely even at location-4 that is almost two duct heights downstream of the grid.
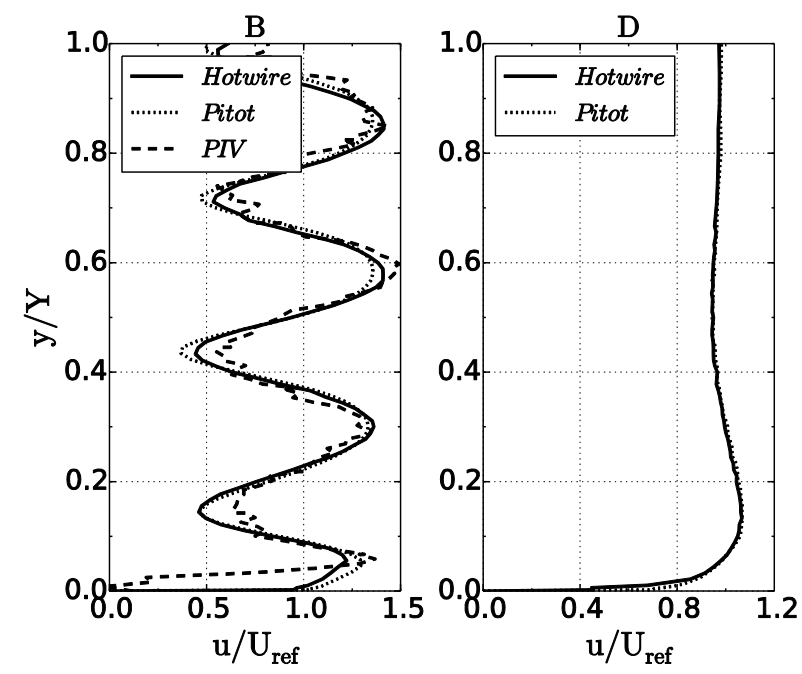

Figure 6. Comparison of velocity profiles from different measurement techniques. B) location-3 and D) location-4. Here $\mathrm{Y}$ corresponds to $\mathrm{y}=45 \mathrm{~mm}$ which is the last traverse coordinate normal to the surface. 
Different measurement techniques such as hotwire, PIV and flattened pitot are being used in this study and they provide results that are consistent with one another as shown in Figure 6. As mentioned elsewhere, the PIV measurements are currently incomplete.

Table 2. Comparison of freestream flow parameters; measured vs Roach's correlation.

\begin{tabular}{|l|l|l|l|l|l|l|}
\hline & \multicolumn{2}{|c|}{$K$} & \multicolumn{2}{c|}{ Tu } & \multicolumn{2}{c|}{$\lambda_{x}$} \\
\hline & Roach & Exp. & Roach & Exp. & Roach & Exp. \\
\hline NG & & & & 1.96 & & 11.65 \\
\hline G1 & 2.27 & 1.95 & 2.69 & 2.62 & 4.11 & 5.97 \\
\hline G2 & 4.51 & 3.99 & 5.41 & 4.87 & 6.71 & 4.65 \\
\hline G3 & 2.27 & 1.89 & 4.41 & 4.34 & 5.81 & 4.79 \\
\hline G4 & 1.05 & 0.92 & 3.29 & 3.54 & 4.75 & 5.09 \\
\hline G5 & 2.27 & 2.31 & 7.23 & 7.87 & 8.22 & 5.02 \\
\hline
\end{tabular}

A comparison of the freestream derived pressure drop coefficient (between location-1 and location-4) and turbulence intensity and length-scale data for the measurements are shown in Table 2. The measured pressure drop coefficient $(K)$ is well in agreement with Roach's correlation for SMS grids. The freestream turbulence values $(\mathrm{Tu})$ also seem to agree quite well with the correlations presented by Roach. The present measurements provide a way of separating the effects of porosity from that of mesh size variation. For a fixed unit mesh size of $12 \mathrm{~mm}$, increasing the porosity (as seen from grids G2, G3 and G4) results in a reduction in turbulence intensity. The measured length-scales $\left(\lambda_{x}\right)$ on the other hand are seen to increase with porosity. This tells us that, to a first order, the turbulence intensity scales with the strut width and the length-scales scale with the opening size (unlike what Roach's correlation shows). The decay and growth respectively of the freestream turbulence intensity and length-scale at locations 1,3 and 4 are shown in Figures 7 and 8 respectively.

The freestream turbulence intensity (Figures 7) is seen to increase through the grid first (measured between locations 1 and 3 ) and then decay from location-3 to location-4. Although not shown here, the decay rate follows Roach's correlations adequately. The lengthscales (Figure 8) are first seen to reduce as the flow goes past the grid and then to increase further downstream, as expected. The freestream values of turbulence intensity and length-scale are also tabulated in Table 3 for the three measurement locations 1,3 and 4. Also shown is the boundary layer heights $\left(\delta_{99}\right)$ at these locations.

The turbulence intensity and length-scale variation at a height corresponding to $33 \%$ boundary layer edge velocity is plotted in Figures 7 and 8 respectively. For unperturbed velocity profiles the boundary layer edge velocity is the same as freestream velocity whereas for profiles with an overshoot the maximum velocity point is taken as the boundary layer edge. It is quite apparent that the strong coupling between porosity and the variation of the above parameters is non-existent. This suggest that there are different physical mechanism at work within the boundary layer such as an increased level of vorticity, in relation to the freestream flow, that could be causing a decoupling of local grid geometric parameters and the growth or decay of turbulence in this region. The values of turbulence intensity and length-scale at $33 \%$ boundary layer height is also tabulated in Table 4.

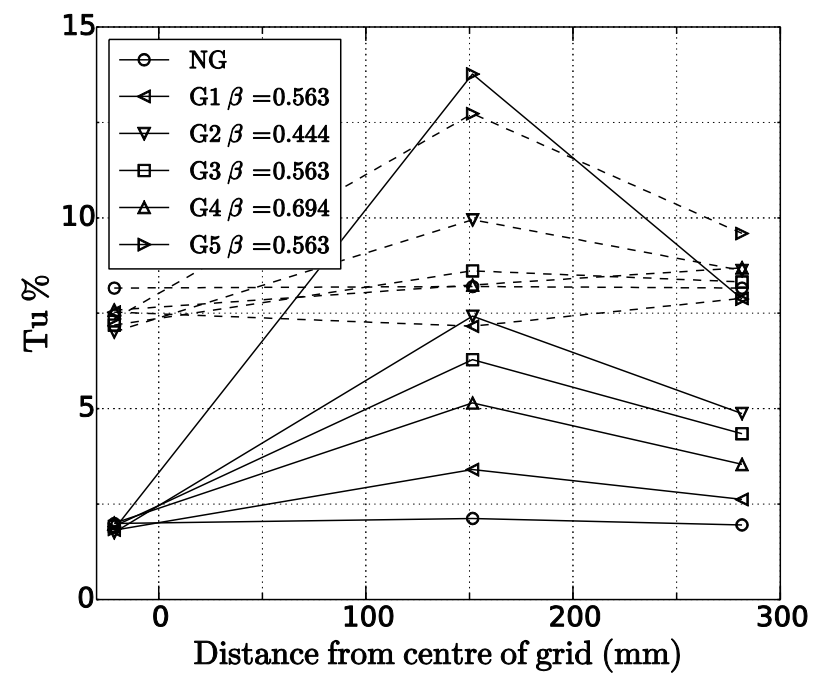

Figure 7. Turbulence decay with distance downstream of the grid: solid lines show freestream variation, dashed lines show variation inside boundary layer at $33 \%$ velocity location.

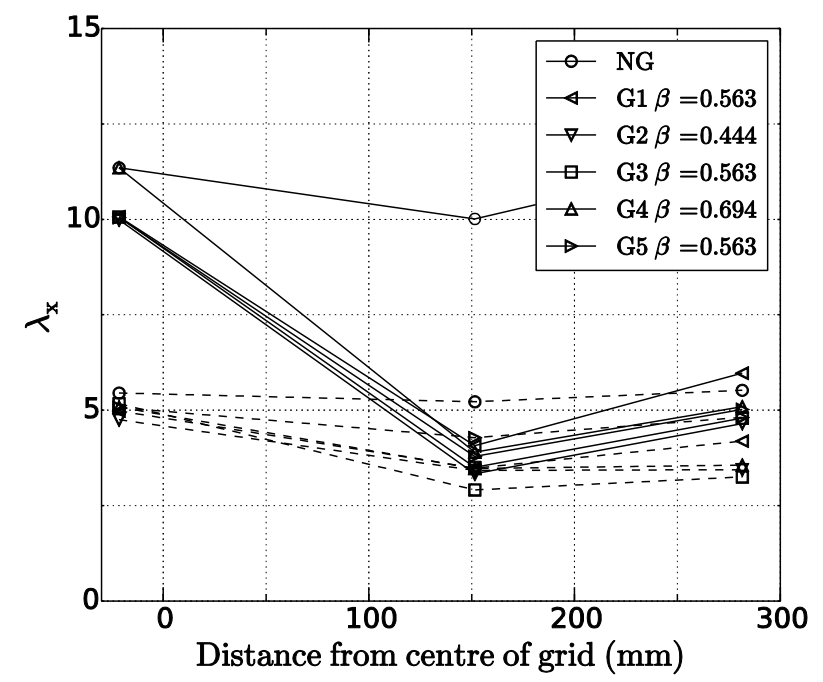

Figure 8. Turbulent length-scale growth with distance downstream of the grid: solid lines show freestream variation, dashed lines show variation inside boundary layer at $33 \%$ velocity location.

When the porosity is fixed and the unit mesh size is varied, the measured turbulence intensity values have been observed to increase with an increase in mesh size. This therefore scales with the strut size. However the measured length-scale values didn't seem to increase as per the correlations and as with the previous observation in the paper that this scales with the mesh opening size. This latter result needs to be revisited as the measurement of length-scales has proven difficult in some of the experiments due to the presence of a small bump in the spectra in the very low frequency region. No immediate source could be identified as a reason for its existence. The results however looked promising overall. 
Table 3. Freestream turbulent flow statistics.

\begin{tabular}{|l|l|l|l|l|l|l|}
\hline & NG & G1 & G2 & G3 & G4 & G5 \\
\hline \multicolumn{7}{|c|}{$\delta_{99}$} \\
\hline Loc-1 & 15.5 & 13.3 & 10.4 & 11.8 & 13.1 & 9.8 \\
\hline Loc-3 & 18.5 & 14.6 & 4.0 & 4.3 & 5.5 & 3.2 \\
\hline Loc-4 & 19 & 14.1 & 5.3 & 5.5 & 6.5 & 5.5 \\
\hline \multicolumn{7}{|c|}{ Tu } \\
\hline Loc-1 & 1.99 & 1.82 & 1.76 & 1.92 & 1.99 & 1.84 \\
\hline Loc-3 & 2.12 & 3.41 & 7.42 & 6.28 & 5.15 & 9.45 \\
\hline Loc-4 & 1.96 & 2.62 & 4.87 & 4.34 & 3.54 & 7.87 \\
\hline \multicolumn{7}{|c|}{$\lambda_{x}$} \\
\hline Loc-1 & 11.35 & 10.1 & 9.99 & 10.1 & 11.4 & 10.1 \\
\hline Loc-3 & 10.01 & 4.07 & 3.33 & 3.5 & 3.19 & 3.79 \\
\hline Loc-4 & 11.65 & 5.97 & 4.65 & 4.79 & 5.09 & 5.02 \\
\hline
\end{tabular}

Table 4. Turbulent flow statistics inside the boundary layer at $33 \%$ velocity location.

\begin{tabular}{|l|l|l|l|l|l|l|}
\hline & NG & G1 & G2 & G3 & G4 & G5 \\
\hline \multicolumn{7}{|c|}{$T u$} \\
\hline Loc-1 & 8.16 & 7.53 & 7.01 & 7.19 & 7.58 & 7.35 \\
\hline Loc-3 & 8.2 & 7.16 & 9.95 & 8.61 & 8.24 & 12.8 \\
\hline Loc-4 & 8.16 & 7.89 & 8.62 & 8.32 & 8.69 & 9.59 \\
\hline \multicolumn{7}{|c|}{$\lambda_{x}$} \\
\hline Loc-1 & 5.45 & 4.98 & 4.75 & 5.17 & 5.08 & 5.07 \\
\hline Loc-3 & 5.22 & 3.48 & 3.42 & 2.91 & 3.47 & 4.28 \\
\hline Loc-4 & 5.52 & 4.19 & 3.44 & 3.25 & 3.56 & 4.81 \\
\hline
\end{tabular}

\section{Conclusions}

A set of experiments have been conducted in a lowspeed wind tunnel with a modified cross section where an interchangeable turbulence grid could be placed. All measurements are conducted at $10.6 \mathrm{~m} / \mathrm{s}$. The flow past the grids is investigated for the nature of the interaction between the grid structure and the incoming boundary layer flow.

All the grids tested produced a pressure drop across it and the pressure drop magnitudes are in good agreement with the correlations for SMS grids by Roach.

For a fixed unit mesh size of $12 \mathrm{~mm}$, varying the porosity has the effect that an increasing porosity produced a reduced level of freestream turbulence but at increased length-scales. This means that the turbulence intensity scales with the strut width and the length-scales scale with the opening size. Roach's correlation however suggests that both these parameters scale with the strut size alone. However, inside the boundary layer both the intensity and length-scale do not seem to have such a strong relationship with the porosity parameter.

A strong coupling has been observed between porosity and the strength of the velocity overshoot, for a fixed mesh size, in that lower the porosity higher is the overshoot found.

Small variations in pressure drop coefficient was observed between meshes of the same nominal porosity but this is very likely due to manufacturing imperfections resulting in local variation of the coefficient of discharge of the individual mesh openings. Turbulence intensity in the freestream has been observed to increase, at fixed porosity, with increased mesh size (therefore with increased strut width) whereas the measured freestream length-scale values did not show a coherent variation. The effect of increased mesh size for a fixed porosity on the overshoot effect is a notable result in that there seems to be an 'optimum' mesh size that generates the largest overshoot. This suggests that the simple way to look at the overshoot as a result of differential pressure drop across the boundary layer is insufficient in explaining the real physical mechanism that is causing the overshoot formation.

The turbulence decay and the length-scale growth in the freestream with distance downstream of the grid appear to follow the correlations of Roach although slightly elevated length scales were observed. The calculation of length-scales needs to be revisited to substantiate the accuracy of some of the findings.

Although the PIV measurements are still ongoing, the averaged results from the various techniques employed for boundary layer investigations (i.e. pneumatic traverse, hotwire traverses and PIV) have looked consistent and they complement each other well in providing physical insight.

\section{References}

1. P.R. Owen, H.K. Zienkiewicz, J. Fluid Mechanics, 2, (1957)

2. Y.L. Lau, W.D. Baines, J. Fluid Mechanics, 33, (1968)

3. R.D. Mehta, AIAA Journal, 23, (1985)

4. P.E. Roach, J. Heat and Fluid Flow, 8 (1986)

5. T. Kurian, J.M. Fransson, Fluid Dynamics Research, 41 (2009)

6. L.A. El-Gabry, D.R. Thurman, P.E. Poinsatte, NASA/TM - 2014-218403 (2014) 